\title{
THE ENVIROMENT, INTERNATIONAL STANDARDS, ASSET HEALTH MANAGEMENT AND CONDITION MONITORING; AN INTEGRATED STRATEGY
}

\author{
S. Roe' ${ }^{1}$ D. Mba $^{2}$ \\ ${ }^{1} \mathrm{CSD}$, British Institute of Non-Destructive Testing (BINDT), UK \\ ${ }^{2}$ School of Engineering, Cranfield University, MK43 0AL, Bedfordshire, UK, \\ Tel: +44-1234754681; Fax +44-1234750728, d.mba@ cranfield.ac.uk
}

\begin{abstract}
Asset Health Management (AHM), supported by condition monitoring (CM) and performance measuring technologies, together with trending, modelling and diagnostic frameworks, is not only critical to the reliability of high-value machines, but also to a companies Overall Equipment Efficiency (OEE), system safety and profitability. In addition these protocols are also critical to the global concern of the environment. Industries involved with monitoring key performances indicators (KPI) to improve OEE would benefit from a standardised qualification and certification scheme for their personnel, particularly if it is based on internationally accepted procedures for the various $\mathrm{CM}$ technologies that also share the same objectives as $\mathrm{AH}$ and $\mathrm{CM}$. Furthermore, the development of 'models' for implementation of a Carbon tax is intrinsically dependent on the integrity and accuracy of measurements contributing to these indicators. This paper reviews the global picture of condition monitoring, the environment and related international standards and then considers their relationship and equivalent global objectives. In addition, it presents the methods behind the development of such standards for certification of competence in personnel involved with data collection, modelling and measurements of KPIs. Two case studies are presented that highlight the integrated strategy in practise.
\end{abstract}

Keywords: Condition monitoring, asset health management, qualification standards, third party certification, acoustic emission. 


\section{Introduction}

The principles of Asset Health Management (AHM) through maintenance protocols (preventive, predictive, reactive, condition-based and reliability-centred), operational procedures and/or generic asset health management protocols are well established. Further, the strategy of continuously monitoring the mechanical and/or material integrity and condition of critical assets (machines, structures, etc), referred to as Condition Monitoring (CM), has been the fundamental backbone of many of these protocols over the last 30 years and includes data collection for Key Performance Indicators (KPIs), evaluation and diagnostics across many industries.

When assessing the condition or health of a given asset, it is normal to understand the expected modes of failure and to set up a monitoring scheme that identifies changes, which are known to lead to the initiation of a known failure mode. The objective of $\mathrm{CM}$ programmes is to ensure safety, optimise the machines efficiency of energy conversion, maximise product yield while minimising energy and product wastage. This is also the primary objective of Overall Equipment Efficiency (OEE) driven industries. Equally, these objectives result in optimised useful life times and improve the assets Whole Life Cycle Cost (WLCC) beyond its design life (see section 4)[14]. CM parameters, their trending and modelling are not only critical to commercial efficiency, profitability and 'Return-on-Investment (ROI), but they are also critical to the global environment, since the challenge to the environment is the waste from inefficient asset and processes in industry. 


\section{Condition monitoring}

The CM technologies used in these maintenance protocols include Vibration analysis (VA), Acoustic Emission (AE), Infrared Thermography (IRT) and lubrication management (LM) [5]. The latter includes Oil and wear debris analysis. More fundamental methods for process performance monitoring (measurement of process parameters) includes pressure, temperature, speed, flow rates, etc, which are often used together with the more advanced methods in large asset maintenance, such as in ships [6,7], petrochemical platforms or manufacturing plants. The use of any of these technologies, singularly or integrated, would be employed to monitor the performance and integrity of an asset in any OEE programme.

\section{The environment}

With regard to standards, the environment, and the current development of CM technologies and their associated International standards (ISO), interest has begun at a time of global relevance. This is vindicated by the acceptance of, and commitment to, the Kyoto protocol by many countries and companies concerned with global warming and their 'carbon footprint', together with the proliferation of engineering and CM standards from the international community (ISO) . The public is also becoming increasingly concerned about global warming, recognising that it is the production of waste material like carbon dioxide and waste energy that is the main cause of this problem. Part of the problem is the demand from society for transportation, heating and refuse disposal. People as individuals can help to limit the stress on the environment by minimising their demand on refuse disposal, energy and material products, thus reducing their carbon footprint; but the majority of carbon loading to the atmosphere is from the burning of 
fossil fuels to supply energy to the power, transport, building and industrial sectors [8]. The latter is of particular concern since much of the world's engineering infrastructure has exceeded, or is in the process of exceeding, its design life. For example, an unfortunate consequence of aged assets, for instance, was the recent leakage of a 'British Petroleum' (BP) stretch of pipeline and the recent closure of one its largest oilfields in the US in August 2006 due to pipeline leakage. What ever the cause, the overall concern is always 'what is the carbon footprint'. To address this concern the UK government has always supported the framework for action outlined by the Kyoto Protocol, through initiatives managed by the Department of Trade and Industry (DTI). The DTI White paper [9] published in February 2003, considers a cross-departmental approach to energy policy and emphasises the importance of climate change considerations in overall energy policy in the UK. Such importance was re-iterated by Stern [10] more recently. The development and implementation of action plans to deliver the emission targets and the monitoring of performance is coordinated by the Sustainable Energy Policy Network (SEPN) at the DTI. There are several government programmes, of which the office of the Climate Change Programme (CCP) [9] at the DTI is one. This office sets out a strategic package of policies and measures across all industry sectors by working in partnership with key stakeholders. The benefits and measures of the CCP include amongst many:
a. Improved energy efficiency
b. Lower costs for businesses
c. Less fuel poverty

These key measures are common to the overall objectives of any condition monitoring maintenance or AHM programme. Further key objectives at the CCP include:

- To stimulate new and more efficient and renewable sources of power generation such as wind power, whereby $10 \%$ of power is to come from these new sources by 2010 . Condition monitoring technologies of VA, LM and $\mathrm{AE}$ are being used to monitor 
operational performance parameters of these wind farms to optimise energy output and reduce public concerns for noise in the environment. AE and VA are leading the research and development of this technology to improve their efficiency even further.

- Improvement of the energy efficiency within buildings as required within the UK building regulations. The new UK Building Regulations for air-tightness of new buildings was published in 2005. The condition monitoring technology of IRT will assist this technology to monitor its performance, but has been used for many years throughout the world to monitor all building types for energy-leakage and damage to structures.

The overall concern of these governmental bodies is Climate change. This climate change results from greenhouse-gas emissions associated with economic activities across a range of industrial and commercial sectors. These emissions are not limited to just carbon dioxide, but also include methane, nitrous oxide, hydrofluorocarbons, perfluorocarbons and sulphur hexafluoride from many industrial waste and effluent streams. The full cost of such emissions to date will only be realised in several years and the impact on the environment, financial and social structures can only be postulated [10]. However, consensus is that the atmospheric loading over the past 20 years is responsible for the environmental changes today. The loading to the atmosphere from today's activities will influence the changes suffered in the next few decades.

What is clear is that the consequence of climate change will become disproportionately more damaging with increasing emissions. Policies proposed to begin to stem the levels of emission include the Carbon budget. This budget has the objective to reduce the participant's carbon footprint. The larger your footprint, the more tax you pay. Whilst the process of meeting locally set targets by individual governments is on-going, there are also international negotiations on 
setting target emission based on climate change, whereby the concepts of 'emissions trading' and 'emissions allocation' are being considered. Many countries are already subject to an emissions allocation, while many companies are participating in emissions trading. This is a carbon budget on an international scale, where carbon credits are allocated for certain activities (e.g., carbon uptake by tree stock owned by the company) to counter balance carbon emissions to yield your carbon footprint.

To help monitor this policy the UK Emissions Trading Scheme (ETS) was launched in April 2002, together with the UK National Allocation Plan (NAP), to support the European Communities Emissions Trading Scheme (EUETS) in 2005. At present companies not only have to strive to meet their emission targets, but also must find ways to budget their carbon load to satisfy the EUETS and pay any outstanding carbon levies. Consequently, the real value of condition monitoring surveillance and testing data within most engineering industries towards improved energy and production efficiency, energy management, and OEE is generally understated or misunderstood. Without a measure of performance and improvement, the final carbon levy debt will be based on inaccurate models that assess the carbon balance in the sustainability loops for each asset.

\section{An integrated strategy}

Information related to production efficiency, production costs, material costs, service costs, energy consumption and losses, waste and life cycle of components and systems and the Whole Life Cycle Cost (WLCC) of asset, and CM and performance test data are input parameters to integrated AHM (IAHM) strategies. Each component in the sustainability loop of each asset within a system is optimised by the efficiency of the associated CM maintenance programme that 
serves it. The WLCC component of such an integrated strategy [4] is a holistic view of "whole life' cost analysis that is 'from the cradle to the grave'. The primary aim of WLCC is to aid capital investment decisions on long-term costs of assets. The KPIs in a WLCC framework includes: maintenance, services, component deterioration rates, asset operational costs, service life of components and ratio of maintenance cost to capital cost; all of which are measured via $\mathrm{CM}$ and diagnostic protocols. $\mathrm{CM}$ actually optimises these parameters and contributes to the 'estimated service life (ESLC) parameter, defined by equation (1):

$\mathrm{ESLC}=\mathrm{RSLC} * \mathrm{~A} * \mathrm{~B} * \mathrm{C} * \mathrm{D} * \mathrm{E} * \mathrm{~F}$

Where RSLC is the 'reference service life' [or design life], $\mathrm{A}=$ material/component factor, $\mathrm{B}=$ design factor, $\mathrm{C}=$ workmanship factor, $\mathrm{D}=$ in use (operator) factor, $\mathrm{E}=$ external environment factor and $\mathrm{F}=$ maintenance factor. Condition monitoring also influences $\mathrm{A}$ and $\mathrm{F}$ factors. This relationship, equation (2), for ESLC is the basic Monte Carlo simulation equation:

$\mathrm{Y}=\mathrm{Y}(\mathrm{o}) * \mathrm{D}(\mathrm{x}) * \mathrm{C}(\mathrm{x}) * \mathrm{Q}(\mathrm{x}) * \mathrm{~B}(\mathrm{x}) * \mathrm{M}(\mathrm{x})$

Which generates a probability distribution for $\mathrm{Y}=\mathrm{ESLC}$ over time (x). In a service life model these parameters are sometimes weighted then normalised to facilitate the calculation of an overall 'remaining service life' using weighted averages. With a Markov processing of these data a condition rating transition probability [4] can be determined, which then interfaces into a WLCC model. With no condition monitoring, the factors A, D and E will dominate the system and they will be much less than optimal. Further, with no CM, an asset would likely suffer increased downtime and breakdowns, reducing the life cycle below its design life. The carbon budget from this less efficient asset would be incorrect if a generalised assumed model was to be 
used, as a higher proportion of energy would be lost and carbon emitted.. The sensitivity on the introduction and level of a Carbon price on exhaustible resources such as oil has been noted [10]. The final integrated strategy developed would be required to assist industry in implementing incremental and step changes in the way we live, in order for 'decarbonisation' of the built environment to be realised permanently. The integrated strategy must also include estimation of embodied carbon and the carbon footprint of all products and services.

\section{International Standards}

The establishment of international technical standards for the CM technologies $[5,11]$ and a conformity standard for the certification of CM personnel who collect and manage the data [12], ensures that the data collected and KPIs measured are accurate and reliable using approved procedures [11-14]. The industries collecting the data would also benefit from a standardised qualification scheme specifying these technical standards.

Over the past decade the international standards community has been developing engineering, condition monitoring and qualification standards that specify the requirements for qualification of CM personnel for $3^{\text {rd }}$ party based certification and $2^{\text {nd }}$ party declaration. This standard is the ISO18436 suite [parts 1 to 7] of standards [11] that specifies the technical requirements in the following parts:

- Part 2- for Vibration Analysis testing

- Part 4 and 5- for lubricant testing [Field and laboratory]

- Part 6 for Acoustic Emission testing

- Part 7 for Thermography testing 
The requirements of the $3^{\text {rd }}$ party certifying body are defined in ISO17024 [14]. In order to facilitate these standards for testing personnel into industry, a $3^{\text {rd }}$ party accredited certifying body is required to manage the process.

\section{The BINDT certification scheme}

The unknown quality of training and lack of confidence in testing personnel competency, with a lack of standardisation for CM personnel, has long been issues of concern in many safety-critical industries. To address this concern, as requested by the UK industry, the British Institute of NonDestructive Testing (BINDT) has developed a certification scheme for CM personnel [5] that is accredited by the UK Accreditation Service [UKAS], that conforms with ISO18436 and ISO17024. BINDT approves examination bodies in accordance with ISO17024 and ISO18436-1, and accredits training bodies in accordance with ISO18436-3. ISO17024 requires a management structure similar to that shown in Figure 1, where:

AE - Acoustic Emission; VA - Vibration analysis; IRT - Infrared thermography; LM lubrication management (Oil / Wear analysis); RTC - Remote Training centre; BSI - British Standards Institute; ATO - Accredited Training Organisation; Technical - Condition Monitoring and Diagnostics Technology committee; CMC -Certification Management Committee 


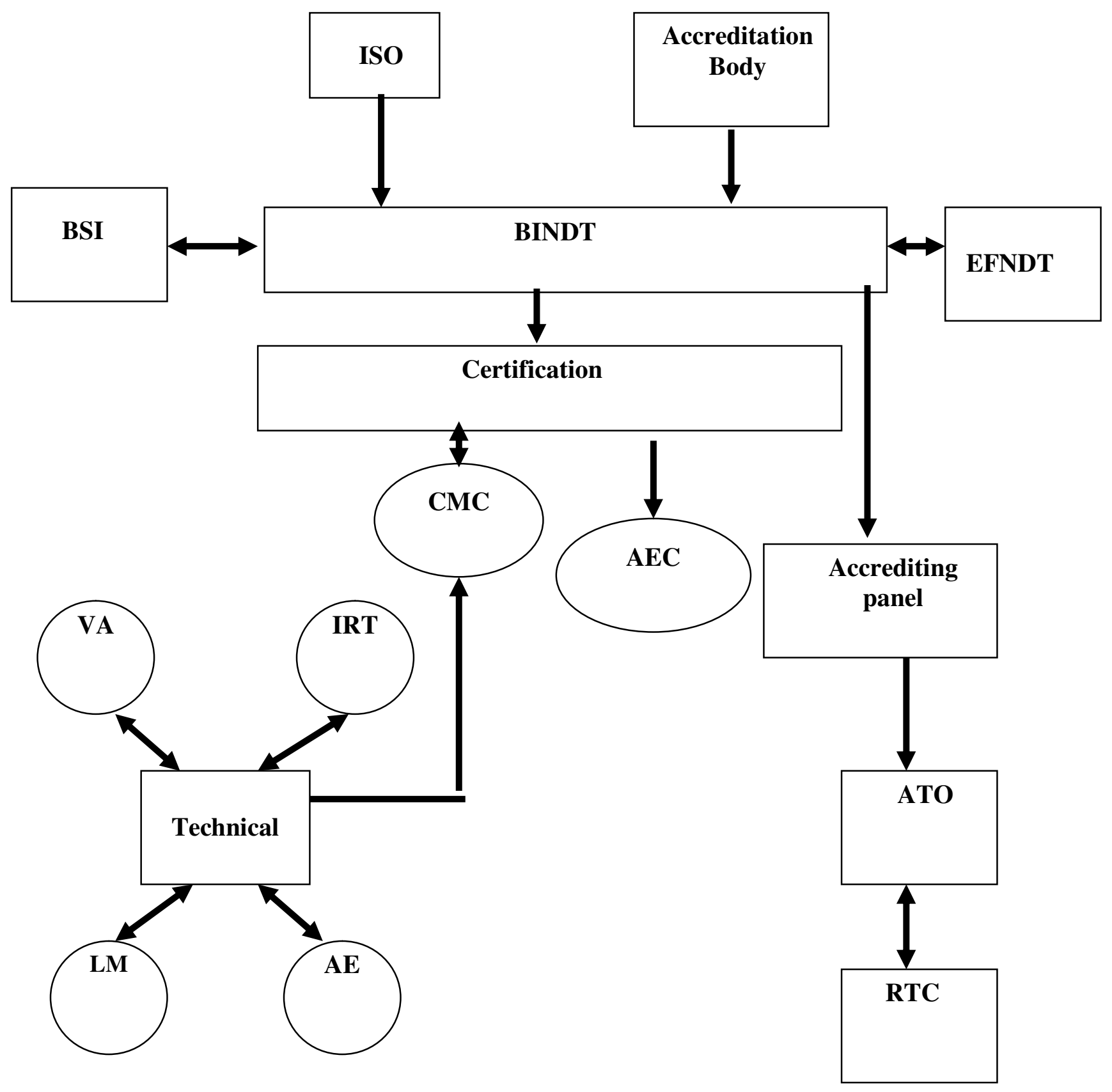

Figure 1 The BINDT CM structure 


\section{Case Studies of Acoustic Emission monitoring in an integrated strategy}

The two case studies presented indicate how a single CM method can contribute to, and influence an AHM outcome. The integration of multi-CM methods and various performance monitoring variable (pressure, temperature etc) of the same component or system can be much more powerful in terms of diagnostics and prognostics.

\subsection{Case Study -1}

Pumps are generally used in most of our domestic and industrial applications. In Europe, pumps consume $117 \mathrm{TWH}$ of electricity per annum, while approximately $10 \%$ of all electricity used by industry and commerce account for $58 \mathrm{MT}$ of $\mathrm{CO}_{2}$ emissions [16]. Every pump manufacturer provides specifications and performance characteristic profiles for their equipment under specified conditions. These profiles demonstrate the inter-relationship between discharge capacities, pump head, power and operating efficiency. The ideal operating point for a pump is known as the 'Best Efficiency Point' (BEP) for that specified condition, and the BEP is normally the target OEE for that pump. This is the point where pump capacity and head pressure combine to provide the maximum efficiency of the pump. If the pump operates outside the BEP, not only will its efficiency be compromised, but it will also be subject to increased wear, thus reducing its operational life, consequent residual life, WLCC and OEE. It has already been acknowledged from previous workers that most pumps operate outside their BEP [17]. For example, a recent study [18] on a 2.2MW centrifugal pump showed that the AET could determine the pumps actual BEP, see figure 2. It is interesting to note that for the case study presented (2.2MW pump), vibration measurements also showed minimum levels at the best efficiency point, though not as 
clearly disenable as values associated with AE activity, see figure 2. The significance of this advance in the application of AET to pumps could allow operators to obtain measurable indicators, either on-line or off-line, that directly correlate to performance and efficiency, thereby allowing for maximisation of pump efficiency and decreasing the WLCC and OEE.

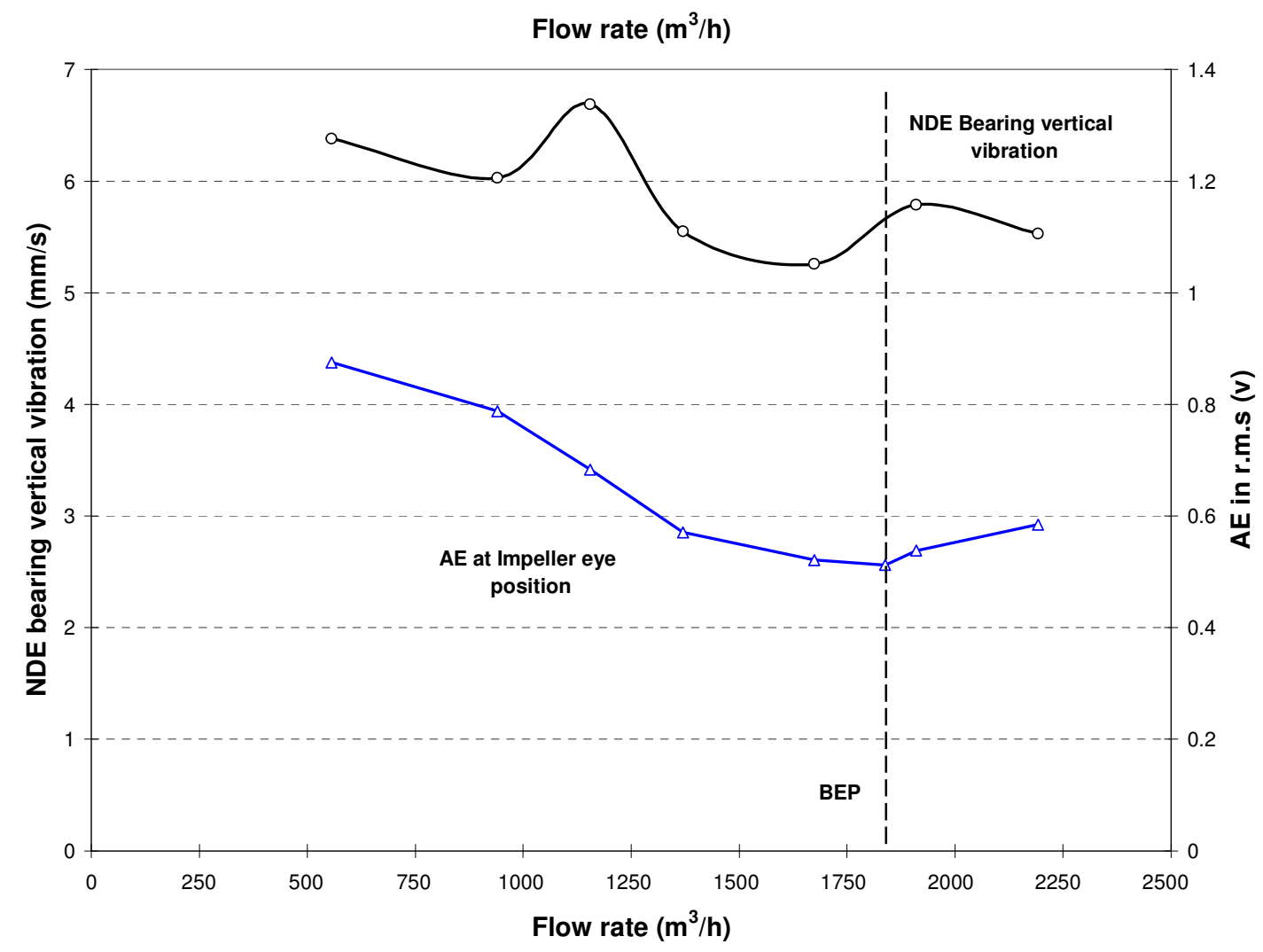

Figure 2 Identification of a pump BEP with the use of a single CM technology [18]

\subsection{Case Study-2}

The following is a case study within the power generation industry of an AE condition monitoring programme where the resulting data is used in a $\mathrm{CM}$ maintenance programme that serves industrial OEE and the environment. It is the application of high frequency Acoustic Emissions Technology (AET) as a means of detecting and rectifying shaft-seal rubbing on an 
operational steam turbine unit (217MVA), to maintain efficiency levels and prolong design life [15]. In the power generation industry rubbing is an undesired contact between a rotating part (main shaft) and stationary part (seal). It usually occurs as a secondary effect of some machine malfunction such as unbalance, misalignment or thermal expansion. Rubbing in large steam turbines is a common occurrence; however, there are occasions when the rubbing becomes severe enough for the machine to be removed from service. This has significant consequences on the WLCC and OEE factors of the AHM for this machine.

The most common method of diagnosing shaft-seal rubbing is vibration monitoring of the bearing pedestals using accelerometers and velocity transducers. However, the use of vibration monitoring only confirms that rubbing is taking place and in some instances, the damage suffered is significant before a measurable change in vibration level is recorded. The generation of $\mathrm{AE}$ during the sliding motion of two mating surfaces is attributed to adhesion, contact and deformation of asperities and the ploughing action of wear particles [18] and it has been shown that the strength and rate of $\mathrm{AE}$ activity is dependent on sliding velocity, friction coefficient of mating surfaces, contact pressure and the height of surface roughness. For these reasons AET is employed for earlier detection of machine malfunction, such as rubbing. Due to its high sensitivity to frictional contact, AET offers an opportunity to detect and locate rotor-stator rubs. More importantly, it allows for the detection of early/incipient rubbing. During operation (3000rpm) of a power generation unit of this case study, measured AE activity showed evidence of rubbing, evident by the presences of periodic AE bursts noted in the waveform, see figure 3 . 

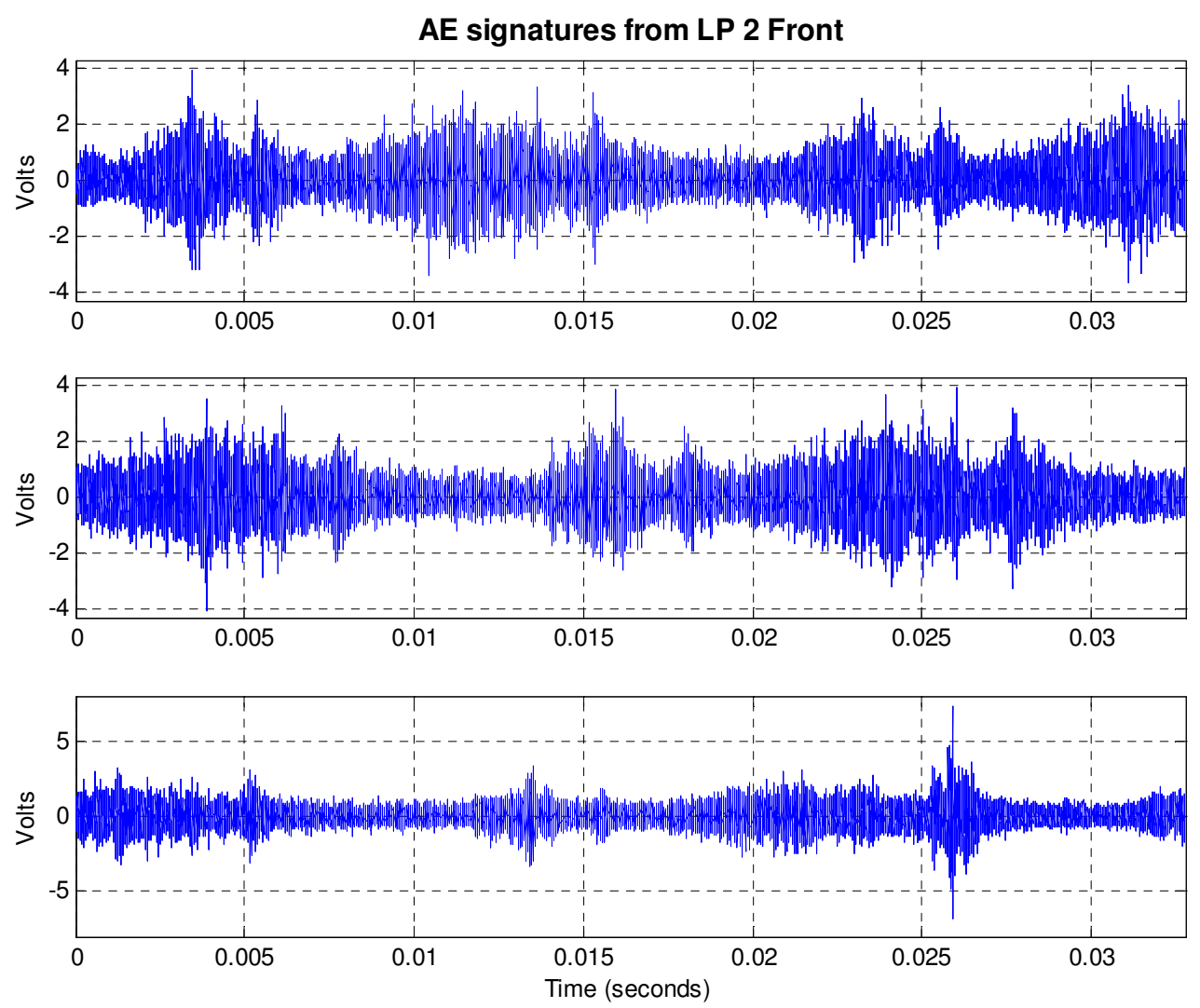

Figure 3 AE waveforms taken from three bearings of an operational turbine unit [15]

Consequently the operating conditions of the turbine units were adjusted to reduce the dynamic shaft displacement to acceptable operational levels. This was achieved by reducing the system vacuum, thereby causing a rise in steam exhaust temperature and thus an increase in clearance between the seals and the rotor. Acoustic Emission was again measured after this change in process condition, with the absence of $\mathrm{AE}$ levels due to the rubbing. This study verified that a singular AE monitoring technology contributes to improvement in WLCC and OEE.

These two case studies exemplify the role that a single CM method can play in decreasing these AHM factors, also reducing environmental emissions by maintaining optimum efficiency levels of operational asset. The significance of an integrated AHM using multi-CM methods and 
performance monitoring tools is obvious. Certification of personnel involved with such measurements to international standards cannot be over emphasised. Further, numerous examples of the application of AET to improving WLCC are publicly available [19] as is the case with other CM technologies.

\section{Conclusions}

Asset Health Management (AHM), supported by condition monitoring (CM) and performance testing technologies, together with trending, modelling and diagnostic frameworks, is not only critical to the reliability of high-value machines, but also to a companies the Overall Equipment Efficiency (OEE), system safety, profitability and 'return on investment' (ROI). AHM and CM are also critical to the global environment and economic sustainable development. Industries that monitor Key Performances Indicators (KPI) to improve OEE and safety as part of an AHM protocol, would benefit from testing personnel who are certified as competent and conforming to specialised ISO qualification standards that share the same objectives as AHM, CM and sustainable development. Furthermore, the development of 'models' and integration strategies for determining Carbon tax burdens imposed by government initiatives is fundamentally dependent on the integrity and accuracy of the AHM data collected. The BINDT scheme presented in this paper shares the same over-riding objectives of safety and OEE as specified for AHM and CM protocols. The certified personnel should apply standards (ISO, EN) that are specific to the engineering asset, where best practice demands. The qualification standards however are best implemented by a certification body offering a $3^{\text {rd }}$ party certification programme accredited in conformity with an international standard. 


\section{REFERENCES}

[1] ISO 15686-1 Service life planning- general principles

[2] R Hooper, K Bourke, W A Ferguson and M Clift, 'An integrated approach to durability assessment throughout the construction procurement', $9^{\text {th }}$ DBMC conference, New Zealand, 2002, paper 123

[3] K C Quillin, G Somerville, R Hooper and P Nixon, 'A framework for service life design of concrete structures', $9^{\text {th }}$ DBMC conference, New Zealand, 2002, paper 128

[4] A Boussabaine and R Kirkham, Whole life-cycle Costing- Risk and risk responses; Blackwell Publishing, 2004

[5] CMGEN, General Requirements for qualification and certification of condition monitoring and diagnostic personnel, UK, issue 2, 2007

[6] Lloyd's Register's (UK), 'ShipRight - Machinery Planned Maintenance and Condition Monitoring, November 2004 - (C) Lloyd's Register, 2004'.

[7] R Hargreaves (VCI), 'Vibration is such a tell-tale'; Maritime IT \& Electronics journal, September 2002

[8] The Sunday Times, Business Focus, Dec $24^{\text {th }}, 2004$.

[9] Climate Change Programme (CCP), DETR, UK, 2003

[10] Stern report, 2006. Cambridge University Press, UK, ISBN 0-521-70080-9).

[11] ISO 18436 'Condition monitoring and diagnostics of machines'; parts 1-7; part 1 (2004), part 2(2003)

[12] EN 473: 2000 -Non destructive testing- Qualification and certification of NDT personnelGeneral principles

[13] ISO 9712: 2005 - Non destructive testing- Qualification and certification of personnel 
[14] BS EN ISO/IEC 17024: 2003- Conformity assessment- general requirements for bodies operating certification of persons

[15] D. Mba, A. Cooke, D. Roby, G. Hewitt, Detection of shaft-seal rubbing in large-scale power generation turbines with Acoustic Emissions; Case study. Journal of power and energy - part A, I Mech E, Vol 218, No. 2, Part A, p 71-82, March 2004. ISSN 09576509.

[16] ETSU and AEAT PLC (2001), Study on improving the energy efficiency of pumps, European Commission report, $\mathrm{p} 70$.

[17] Jantunen, E., Miettinen J and Ollola A,( 1993), Maintenance and downtime costs of centrifugal pumps in Finnish industry, Proceedings of the $13^{\text {th }}$ International Pump technical Conference, London, UK, 9-20.

[18] L. Alfayez, D. Mba, Detection of incipient cavitation and determination of the best efficiency point for centrifugal pumps using Acoustic Emission. Journal of Mechanical Process Engineering, Part E, IMechE, Volume 219 Number 4, November 2005, p 327 - 344.

[19] D. Mba \& Raj B.K.N. Rao, Development of Acoustic Emission Technology for Condition Monitoring and Diagnosis of Rotating Machines; Bearings, Pumps, Gearboxes, Engines and Rotating Structures. The Shock and Vibration digest, 38/1, Jan 2006, 3-16. 